\title{
Adaptive RLS Beamforming for MIMO-OFDM using VBLAST
}

\author{
Shankar Gangaju \\ Department of Electronics and Communication \\ Kathmandu Engineering College \\ Kalimati, Kathmandu, Nepal
}

\author{
Sanjivan Satyal \\ Department of Electronics and Communication \\ Kathmandu Engineering College \\ Kalimati, Kathmandu, Nepal
}

\begin{abstract}
To overcome a multipath fading environment with low complexity and to achieve wireless broadband multimedia communication systems, orthogonal frequency division multiplexing (OFDM) transmission scheme is used. OFDM is one of the applications of a parallel data transmission scheme, which reduces the influence of multipath fading. The performance of OFDM can be improved further using concept of spatial diversity Multiple Input Multiple Output (MIMO). Similarly, Adaptive Beamforming algorithms improve the SNR by focusing antenna patterns on desired angles of reception or transmission, which actually increases the antenna gain. This paper explains the BER performance of MIMO-OFDM system with and without using adaptive beamforming algorithms and both using V-BLAST as the detection scheme. Different modulations are used in Rayleigh fading environment to observe the BER performance by simulations. Furthermore, paper study also shows that RLS beamforming algorithm shows the better performance and is explain by simulations that include array factor plot as well as mean square error plot.
\end{abstract}

\section{Keywords}

Beamforming, MIMO-OFDM, Least Mean Square (LMS), Recursive Least Square (RLS), V-BLAST Zero Forcing, Minimum Mean Square Error(MMSE), Rayleigh, 16-QAM, QPSK

\section{INTRODUCTION}

The new advanced mobile communication systems are expected to provide high quality and high data rate multimedia packet transmission rather than voice-oriented legacy wireless systems. To achieve these innovative features into reality, capability of high spectral efficiency over limited frequency resource with limited transmission power is strongly required. As a challenging technique, the MIMO (Multiple Input Multiple Output) system equipped with sophisticated space-time signal processing algorithms such MMSE (Minimum Mean Squared Error) Zero Forcing (ZF) receiver combined with BLAST architecture [1].

Further, in order to increase spectral efficiency and to effectively overcome deterioting effects of ISI (Inter Symbol Interference) and frequency Selective fading by multipath, MIMO can be combined with OFDM (Orthogonal Frequency Division Multiplexing) system. OFDM is a multicarrier transmission technique that has been recently recognized as an excellent method for high speed bi-directional wireless data communication and its signal offers an advantage in a channel that has a frequency selective fading. Recently Beam formers have drawn much attraction because of its capability of de- correlating spatially distinct channels. It has been already revealed that the Beam formers results in plausible performance under correlated MIMO channels provided that the average channel state information is available on the transmitter. Adaptive beamforming techniques have been employed to enhance desired signal while suppressing the interference, the jammer and the noise in an array of sensors [1], [2].

The paper focuses on the implementation of beamforming in MIMO-OFDM system. Similarly, the paper also explains the different VBLAST detection algorithms under Rayleigh environment with various modulation techniques. The rest of the paper organized as fallows. Section 2 introduces the system descriptions. Brief important theories were explained in section 3, 45 and 6. Performance is compared in various aspects in section 7 . This paper concludes with the section 8 .

\section{SYSTEM DESCRIPTIONS}

First, generate a binary data stream in random manner. This generated data stream is convolutional encoded so that at the receiver side it can be decoded with less bit error rate. The encoded bits are modulated by QPSK/16 QAM then preprocess for the OFDM system. The modulated data are converted into parallel data stream and then added a cyclic prefix so that the Inter-symbol Interference (ISI) can be minimized between OFDM symbols also the Guard Interval is also created so that the Inter Carrier Interference (ICI) between OFDM subcarrier is minimized. Now it is converted into time domain by using Inverse Fast Fourier Transform (IFFT) since the channel can detect the time domain signal. Further the time domain signal are passed through an Eigen beamforming which creates a steering signal to the desired direction which makes a signal with more directive in desired direction. The signals are then passed through Rayleigh fading channel. At the receiver side, the antennas with beam former processed signal are passed to Fast Fourier Transform (FFT) and then remove cyclic prefix and then passed to the $\mathrm{V}$ BLAST detector with ZF/MMSE with channel estimate of RLS and LMS. The detected signal is passed to the parallel to serial converter and demodulated. Finally, signal are decoded by Viterbi decoder and compare the bit that are transmitted and bit that are received. The proposed system model is shown in figure below: 


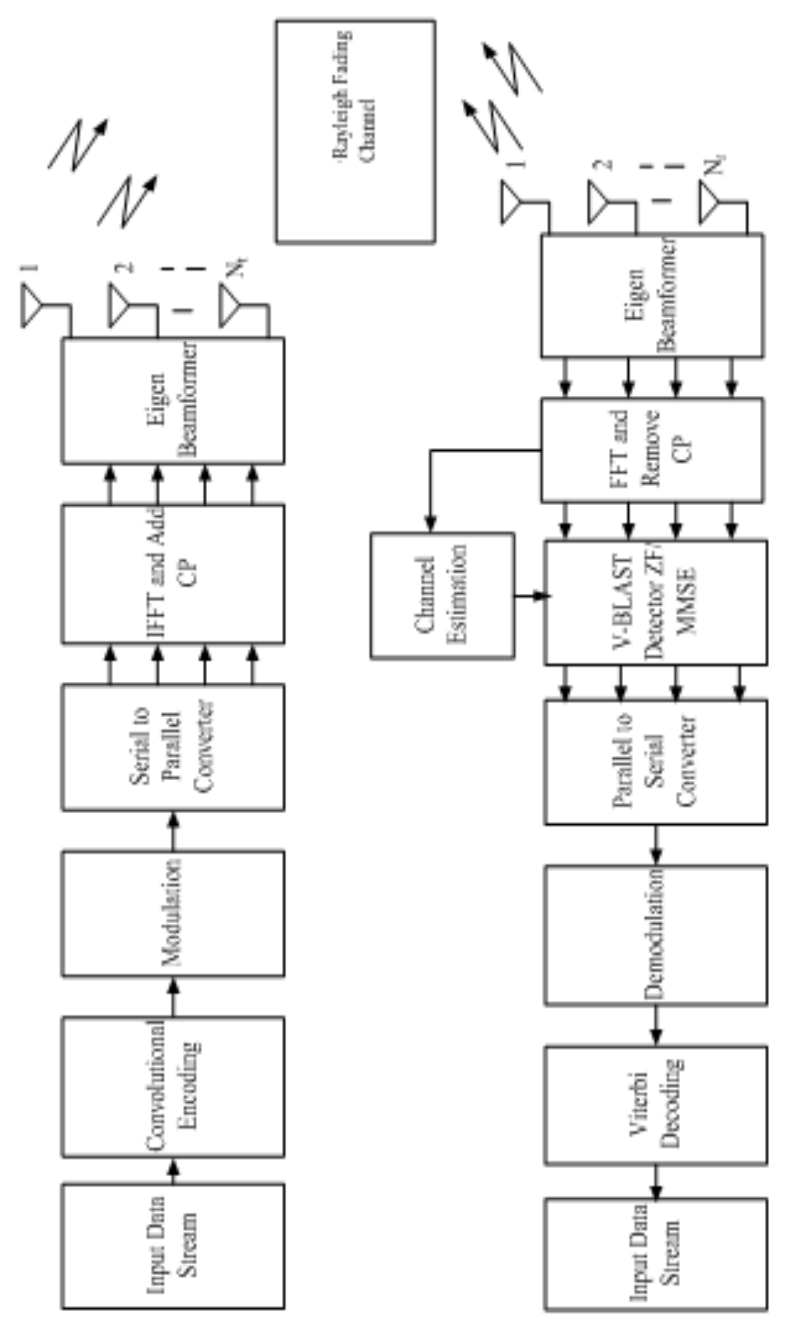

Fig 1: Block Diagram of Proposed System

\section{MIMO}

MIMO can be categorized into three different areas: precoding, spatial multiplexing, and diversity coding. Precoding refers to the spatial processing that is performed at the transmitter (also known as beamforming). The goal of spatial processing is to reduce the effect of multipath fading from constructive interference of the signals being transmitted. Multipath is a propagation phenomenon that is characterized by the arrival of multiple versions of the same signal from different locations shifted in time due to having taken different transmission paths of varying lengths. Precoding is used when a receiver consists of more than one antenna and beamforming cannot maximize the signal level across all receiving antennas. In addition, precoding requires that the transmitter has knowledge of the CSI. Spatial multiplexing gain refers to using the degrees of freedom in a communication system by sending independent symbols in parallel over multiple spatial channels. This technique is a powerful one for increasing channel capacity for higher SNRs and can be used in either a closed loop system or an open loop system. Diversity coding requires a single stream of data to be encoded with a space- time code prior to transmission. Spacetime codes create orthogonality among the data being sent to the receiver. No CSI is required to be known at the transmitter to take advantage of this mechanism of MIMO. The diversity order of a MIMO transmission system over an independent and identically distributed (i.i.d) Rayleigh channel with $n_{t}$ transmit antennas and $n_{r}$ receives antennas at high $\mathrm{SNR}$ is given by:

$$
n_{r}-n_{t}+1
$$

Thus, the diversity order can be improved by increasing the number of receive antennas and can be degraded by increasing the number of transmit antennas. However, increasing the number of transmit antennas increases spatial multiplexing gain so there is generally a trade of made in the design process. There are different architectures used for MIMO communications that can be used when trying to take advantage of this technology. Bell Labs Layered Space Time Architecture (BLAST), Per Antenna Rate Control (PARC), and Selective per Antenna Rate Control (SPARC) are among the most notable. Spatial multiplexing causes the complexity of receiver to drastically increase and as a result techniques such as OFDM are used to handle the multipath channel. The basic MIMO channel model is shown in below [3].

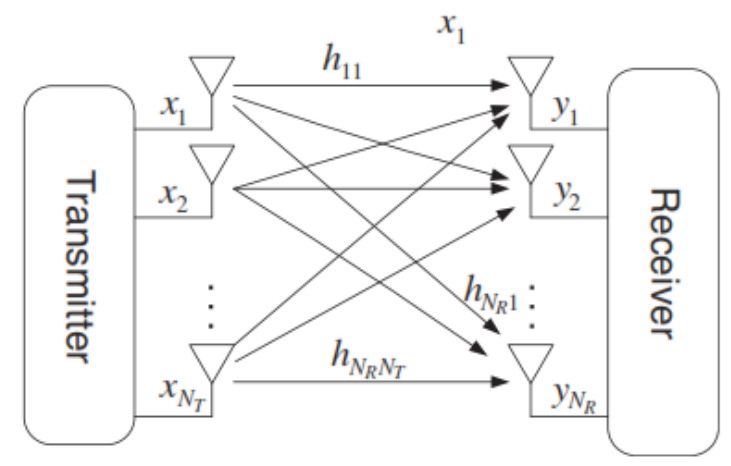

Fig2: MIMO channel model

The transmitter sends several streams of data from multiple antennas. The number of possible paths that the channel will allow is $n_{t}, n_{r}$ where $n$ is the number of transmit antennas and $n$ $r$ is the number of receiver antennas. It is the receiver's job to decode the information received and output the original data set. A MIMO system can be modeled

$$
y=\boldsymbol{H} x+n
$$

Where $y$ is the received vector, $\mathrm{x}$ is the transmitted vector, $\mathrm{n}$ is the noise vector, and $\mathrm{H}$ is the channel matrix.

\section{OFDM}

The OFDM system typically consists of convolution encoder, modulation, and inverse fast fourier transform (IFFT), injection of guard interval (GI).

An OFDM signal consists of $\mathrm{N}$ orthogonal subcarriers modulated by $\mathrm{N}$ parallel data streams.

Each baseband subcarrier is of the form,

$\Phi_{k}(t)=e^{j 2 \Pi f_{k} t}$

Where, $\mathrm{f}_{\mathrm{k}}$ is the frequency of the $\mathrm{k}^{\text {th }}$ subcarrier. One baseband OFDM symbol multiplexes $\mathrm{N}$ modulatedsubcarriers.

$\frac{1}{\mathrm{~S}(t)=} \sum_{k=0}^{N-1} x_{k} \Phi_{k}(t) \underset{0<\mathrm{t}<\mathrm{NT},}{ }$ 
Where $\mathrm{x}_{\mathrm{k}}$ is the $\mathrm{k}^{\text {th }}$ complex data symbol (typically taken from a QAM symbol constellation) and NT is the length of the OFDM symbol.

The subcarrier frequencies $f_{k}$ are equally spaced.

$\mathrm{f}_{\mathrm{k}}=\frac{k}{N T}$, which makes the subcarriers $\Phi_{\mathrm{k}}(\mathrm{t})$ on $0<\mathrm{t}<\mathrm{NT}$, orthogonal. The signal separates data symbols in frequency by overlapping subcarriers, thus using the available spectrum in an efficient way [4], [5].

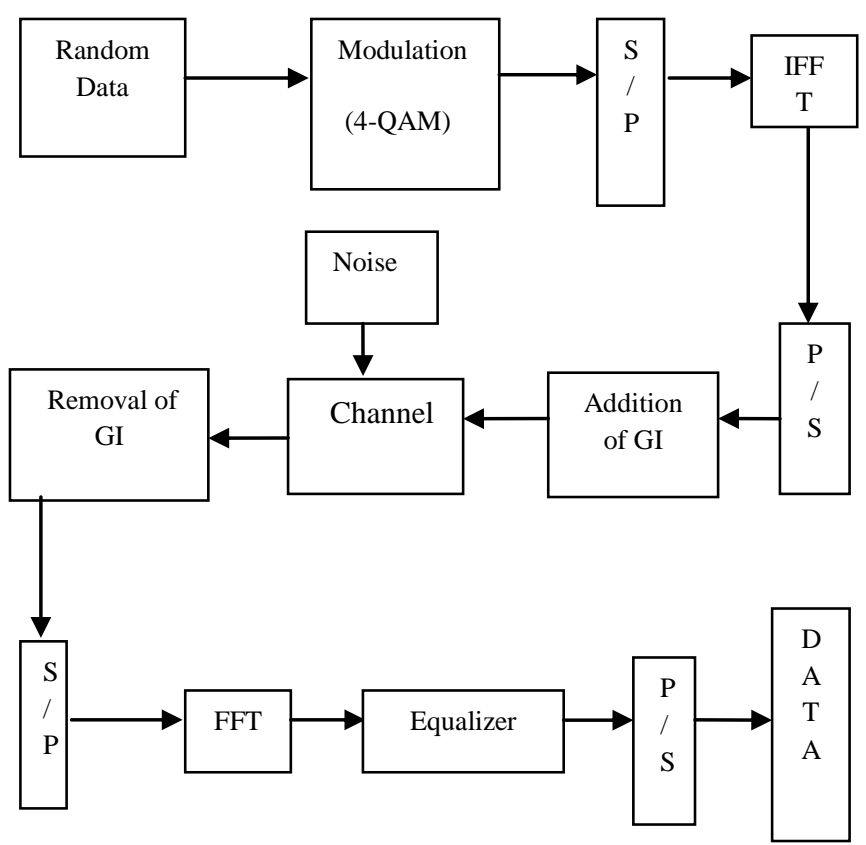

Fig 3: OFDM System Description

\section{V-BLAST}

The layers are arranged horizontally across space and time for V-BLAST and the cycling operation is removed before transmission. At the receiver side, each receive antenna is a superposition of $\mathrm{M}$ faded symbols plus Additive White Gaussian Noise (AWGN).

Successive Interference Cancellation (SIC) is incorporated with V-BLAST architecture for both ZF and MMSE detection algorithm so as to improve the performance of detection algorithm. The detection process consists of two main operations.

a. Interference Suppression (nulling): The interference is null out by projecting the received vector onto the null subspace (perpendicular subspace) of the subspace by the interfering signals. After that, normal detection of the first symbol performed.

b. Interference Cancellation (Subtraction): The contribution of the detection is subtracted from the receiver.

V-BLAST requires its operation to be conducted in a multipath environment in order to achieve a sufficient level of de-correlation at the receiver and these conditions allow the receiver to distinguish signals occupying the same channel space. V-BLAST and OFDM can be combined in frequency selective fading channels to achieve a high rate of data transmission [4], [6].

\subsection{V-BLAST ZF}

Zero forcing receivers are a simple linear receiver with low computational complexity. It minimizes interference but suffers from noise enhancement's receiver works best with high SNR level. Zero Forcing implements matrix (Pseudo) inverse (+). The $\mathrm{ZF}$ estimated receive signal is given by:

$$
\hat{X}=\left(H^{H}\right)^{-1} H^{H} \cdot X=H^{+} X
$$

Where the zero forcing decoding matrix is as follows:

$$
S_{Z F}=\left(H^{H} H\right)^{-1} H^{H}
$$

Where superscript $\mathrm{H}$ denotes Hermitian transpose [1]

\subsection{V-BLAST MMSE}

At very high SNR level decorrelator completely suppress the interference, therefore it provide better performance at higher SNR level. Bur in low SNR level condition the maximal ratio combining receiver provide better performance. Therefore in order to design an optimal receiver it is necessary to converge these two advantages in a single receiver. In MMSE receiver this two features are optimally combined. MMSE receiver is another type of linear detector which minimizes the mean squared error between the transmitted symbols. MMSE detector helps to jointly minimized both the noise and interference i.e. MMSE detector seeks to balance between cancellation of the interference and reduction of noise enhancement. Therefore, MMSE detector outperforms the ZF detector in the presence of noise. MMSE receiver gives a solution of:

$$
\hat{X}=\left(\frac{1}{S N R} I+H^{H} H\right)^{-1 .} H^{H} X
$$

The above linear equalization algorithm is based on multiplying the received vector by a detection matrix and then decoding the symbols separately. Another approach in VBLAST receiver design is successive interference cancellation to achieve better performance at the cost of much higher complexity [2], [6].

\section{BEAMFORMING}

Beamforming or spatial filtering is a signal processing technique used in sensor arrays for directional signal transmission or reception. This is achieved by combining elements in a phased array in such a way that signals at particular angles experience constructive interference while others experience destructive interference. Beamforming can be used at both the transmitting and receiving ends in order to achieve spatial selectivity [7].

The improvement compared with omni directional reception/transmission is known as the receive/transmit gain (or loss).Beamforming can be used for radio or sound waves. It has found numerous applications in radar, sonar, seismology, wireless communications, radio astronomy, acoustics, and biomedicine. Adaptive Beamforming is used to detect and estimate the signal-of-interest at the output of a sensor array by means of optimal (e.g., least-squares) spatial filtering and interference rejection [7], [8]. 


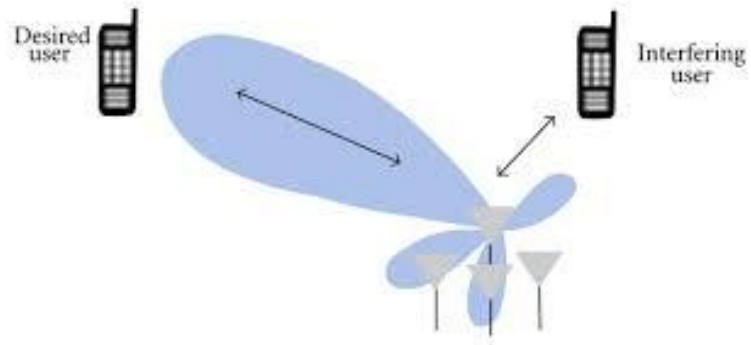

Fig 4: Beamforming

Beamforming techniques can be broadly divided into two categories:

a) conventional (fixed or switched beam) beam formers

b) adaptive beam formers or phased array

Adaptive Beamforming techniques generally combine this information with properties of the signals actually received by the array, typically to improve rejection of unwanted signals from other directions

\subsection{RLS ALGORITHM}

RLS algorithm is a type of Non-blind algorithm because it uses the training signal or reference signal. The RLS weight update equation can be written as

$W(n+1)=W(n)+\alpha(n) * h(n)$

The RLS algorithm is used to reduce the error using the following equation:

$E(p)=\sum_{K=0}^{P} \lambda^{p-k}|e(p)|^{2}=\sum_{K=0}^{P} \lambda^{p-k}\left|d(k)-W_{p}^{H} x_{k}\right|^{2}$

Here $\lambda$ is a constant term having value $\lambda \leq 1$ and so the error term is exponentially weighted toward the recent data samples. The reason for the fast convergence of the RLS is due to the fact that that RLS technique whitens the input data by using the inverse correlation matrix of the data. Despite the initial presentation of the weight update includes the correlation matrix inversion, recursive computations can be employed to update the value of the correlation matrix of the input step by step [9] [10].

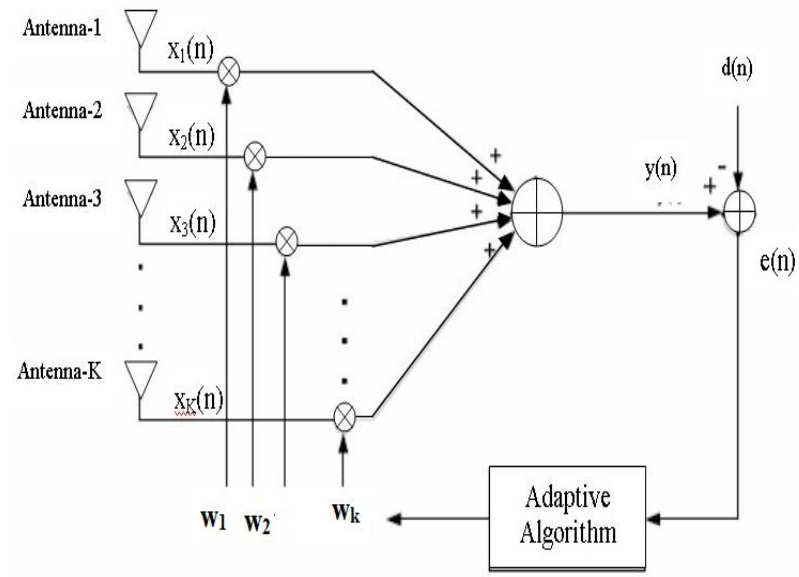

Fig 5: Adaptive Beamforming System.

\section{SIMULATION AND PERFORMANCE ANALYSIS}

\subsection{RLS Algorithm Simulation Parameter}

Table 1: RLS Algorithm Simulation Parameter.

\begin{tabular}{|l|l|}
\hline Desired Signal Angle & $20^{\circ}$ \\
\hline Interference Signal Angle & $60^{\circ}$ \\
\hline SNR in dB & 15 \\
\hline Number of Elements & 8 \\
\hline Data Sampled & 100 \\
\hline Forgotten Factor $(\alpha)$ & 0.99 \\
\hline
\end{tabular}

\subsection{Simulation of RLS Algorithm}

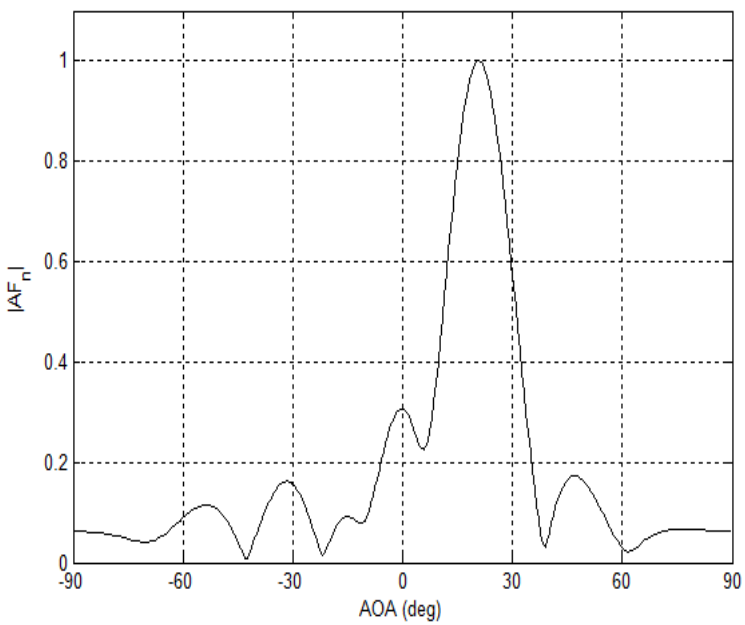

Fig 6: Array Factor Plot

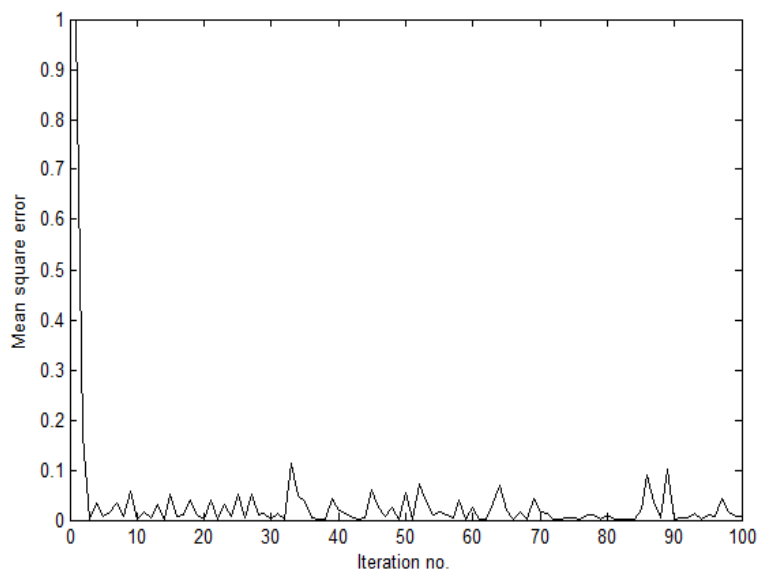

Fig 7: Mean Square Error Plot 
RLS is proposed as the Beamforming algorithm and the number of elements considered for simulation environment is 8.From the figure 6 , it can be explained that RLS algorithm provides deeper nulls in the direction of the interference. The assumed simulation parameters are tabulated in above table 1 . Figure 6 explains the array factor plot where interference signal is completely rejected at 60 degree is shown. It is known that the LMS adaptive algorithm has a slow convergence when the Eigen values of the covariance matrix are widespread and hence the convergence takes much more time [9]. Unlike LMS algorithm, RLS algorithm has fast convergence since matrix Inversion computation is not require in RLS algorithm as the inverse correlation matrix is computed directly. The fastest convergence of RLS is due to the fact that recursive computations can be employed to update the value of the correlation matrix of input step by step. The fastest convergences of RLS algorithm is shown in Figure 7 clearly with mean square error plot.

Table 2: Simulation Parameter Used.

\begin{tabular}{|l|l|}
\hline Parameters & Specification \\
\hline Number of Iteration & $10^{5}$ \\
\hline FFT Size & 64 \\
\hline Guard Interval & $1 / 4$ \\
\hline Number of Carrier & 64 \\
\hline Signal Constellation & QPSK and 16QAM \\
\hline Channel Model & Rayleigh \\
\hline Detection Algorithm & V-BLAST \\
\hline Beamforming Type & Adaptive \\
\hline Beamforming & LMS and RLS \\
\hline
\end{tabular}

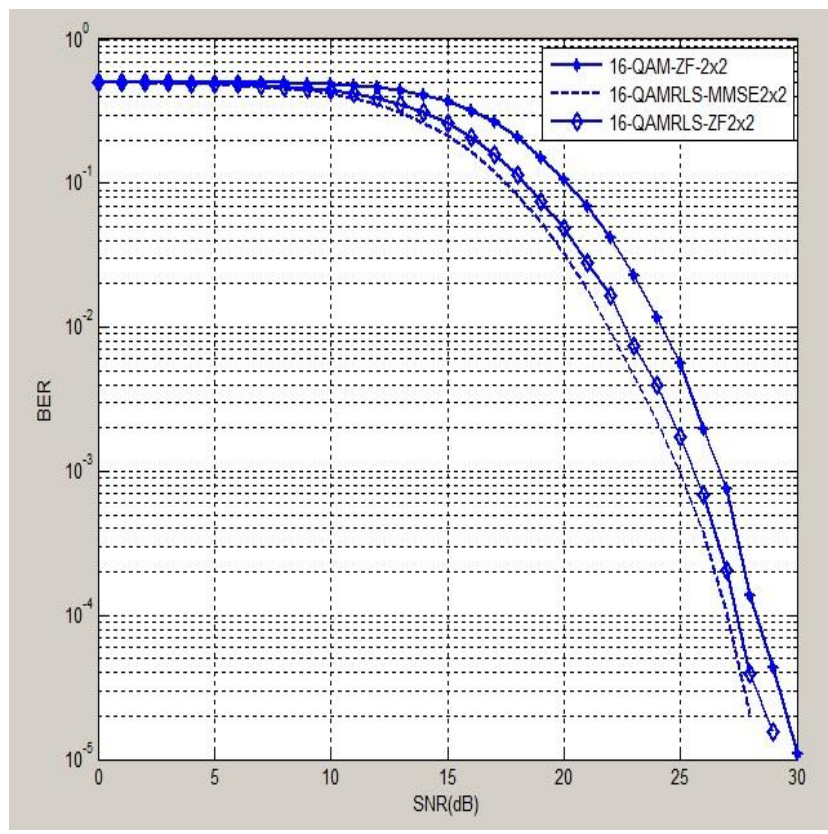

Fig 8: SNR vs. BER for V-BLAST -ZF/MMSE MIMOOFDM with RLS and Without Beamforming for 16QAM using 2x2 Antenna

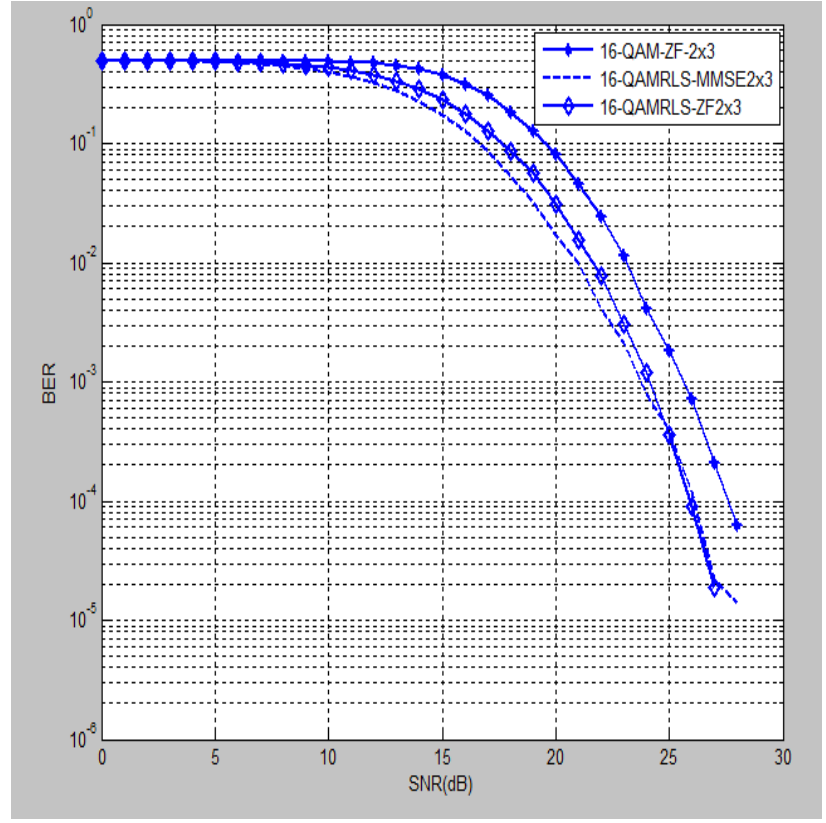

Fig 9: SNR vs. BER for V-BLAST -ZF/MMSE MIMOOFDM with RLS and Without Beamforming for 16QAM using 2x3 Antenna

Figure 8 and Figure 9 show the BER vs. SNR plot for $2 \times 2$ and 2x3 MIMO antenna system for 16QAM respectively. Both figures also explain the VBLAST-ZF/MMSE for MIMO OFDM system with RLS adaptive algorithm and without Beamforming. The results show that MMSE Nulling technique produces the better result over $\mathrm{ZF}$. In figure 8 nearly $2 \mathrm{~dB}$ of SNR value is dropped down for MMSE (at $10^{-3}$ point). Similarly, in figure 9 nearly $2 \mathrm{~dB}$ of SNR value is dropped down for MMSE case and $1 \mathrm{~dB}$ of SNR value is dropped down for $\mathrm{ZF}$ case with $2 \times 3$ antenna configuration (at $10^{-3}$ point )

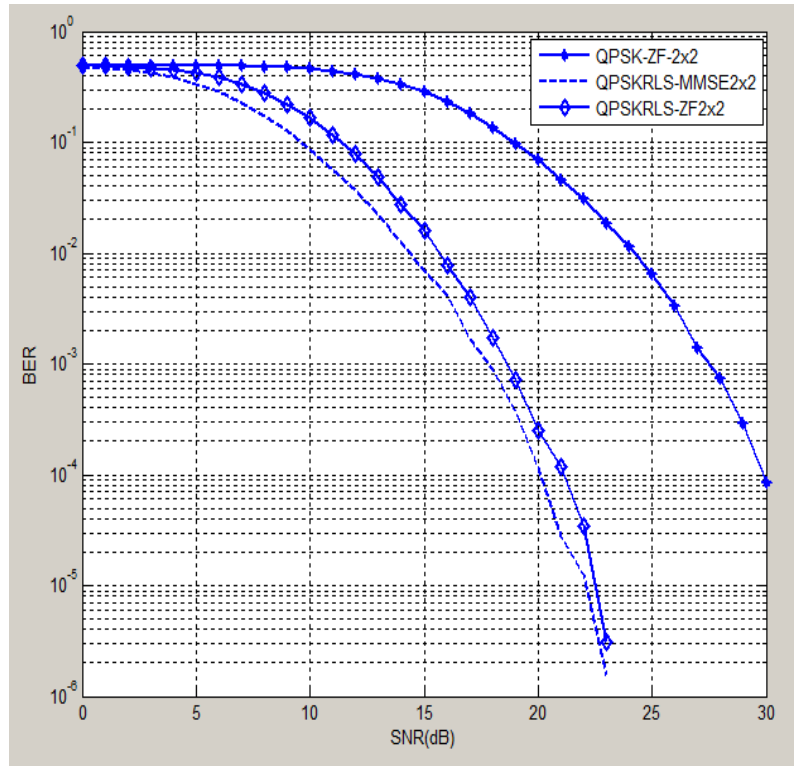

Fig 10: SNR vs. BER for V-BLAST -ZF/MMSE MIMOOFDM with RLS and Without Beamforming for QPSK using 2x2 Antenna 


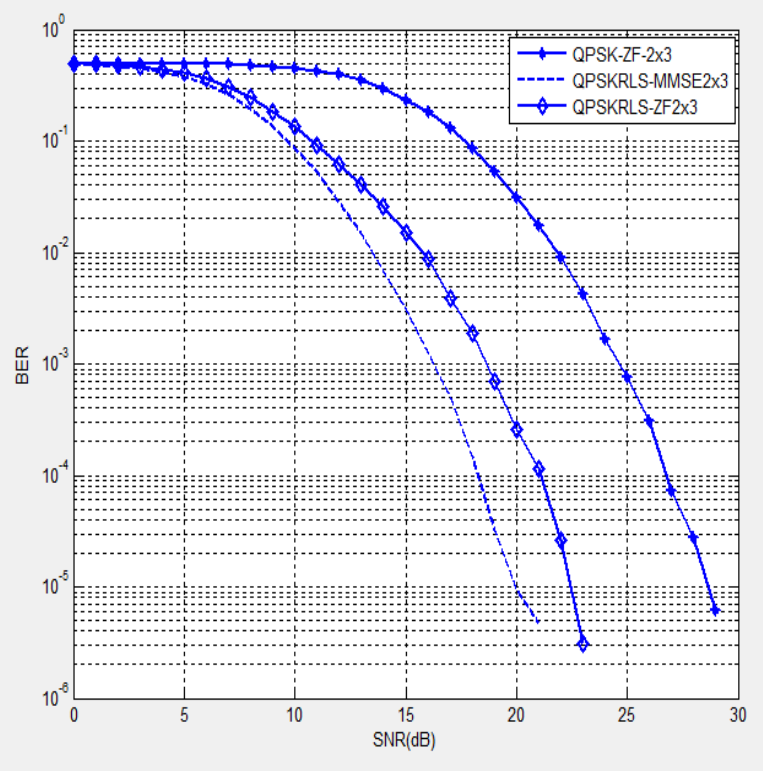

Fig 11: SNR vs. BER for V-BLAST -ZF/MMSE MIMOOFDM with RLS and Without Beamforming for QPSK using $2 \times 3$ Antenna

Figure 10 and Figure 11 show the BER vs. SNR plot for $2 \times 2$ and 2x3 MIMO antenna system for QPSK respectively. Both figures also explain the VBLAST-ZF/MMSE for MIMO OFDM system with RLS adaptive algorithm and without Beamforming. The results show that MMSE Nulling technique produces the better result over ZF. In Figure 10 nearly $10 \mathrm{~dB}$ of SNR value is dropped down for MMSE case and $9 \mathrm{~dB}$ of SNR value is dropped down for ZF case with $2 \times 2$ antenna configurations $\left(10^{-3}\right.$ point). Nearly $8 \mathrm{~dB}$ of SNR value is dropped down for MMSE case and $5 \mathrm{~dB}$ of SNR value is dropped down for $\mathrm{ZF}$ case with $2 \times 3$ antenna configuration $\left(10^{-3}\right.$ point $)$ is shown in Figure 11

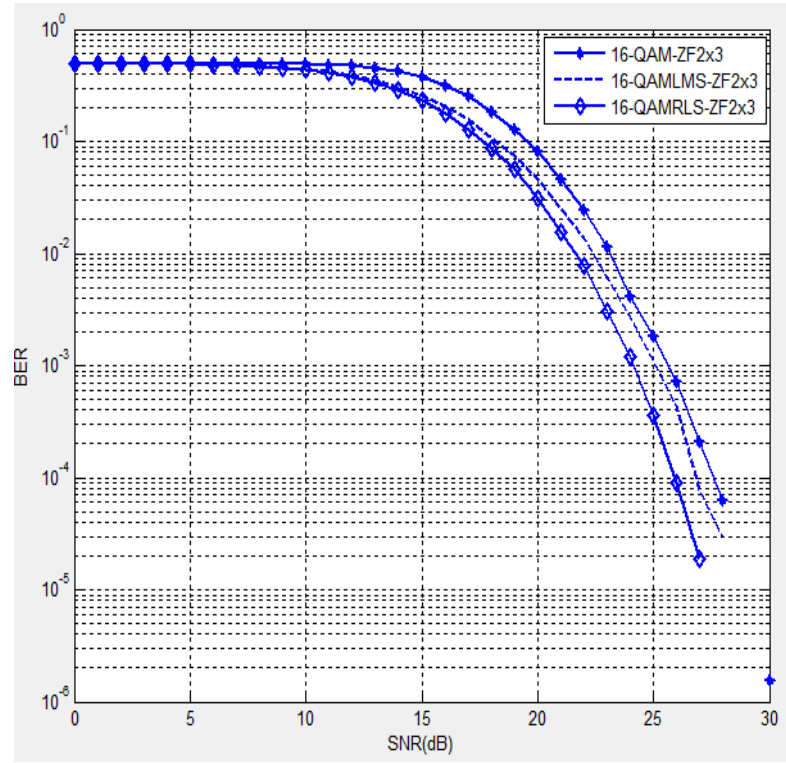

Fig 12: BER analysis for V-BLAST-ZF MIMO-OFDM for with (RLS \& LMS) and Without Beamforming for 16QAM using 2X3 Antenna.

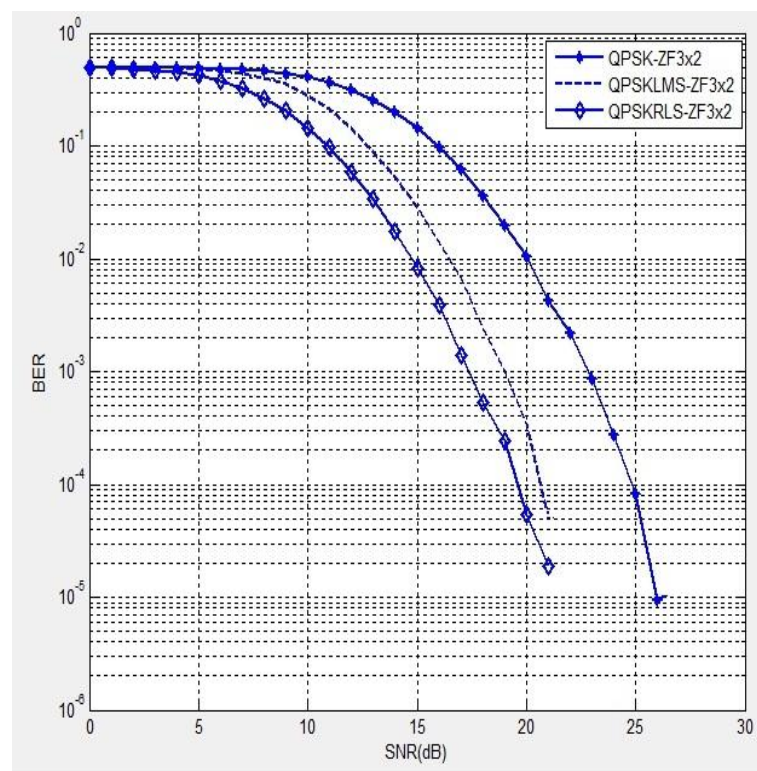

Fig 13: SNR vs. BER for V-BLAST-ZF MIMO-OFDM for with (RLS \& LMS) an Without Beamforming for QPSK using 3X2 Antenna.

Figure 12 and Figure 13 shows the BER vs. SNR plot for $2 \mathrm{X} 3$ and 3X2 MIMO-OFDM antenna configuration respectively under the 16-QAM and QPSK Modulation techniques. VBLAST-ZF is used as the detection algorithm in both cases. Simulations explain the performance of MIMO-OFDM system using and without using Adaptive beamforming algorithms. It is observed that MIMO-OFDM system has better performance when adaptive algorithms are used. Also the RLS algorithm outperforms over the LMS algorithm in both 16-QAM and QPSK modulation techniques.

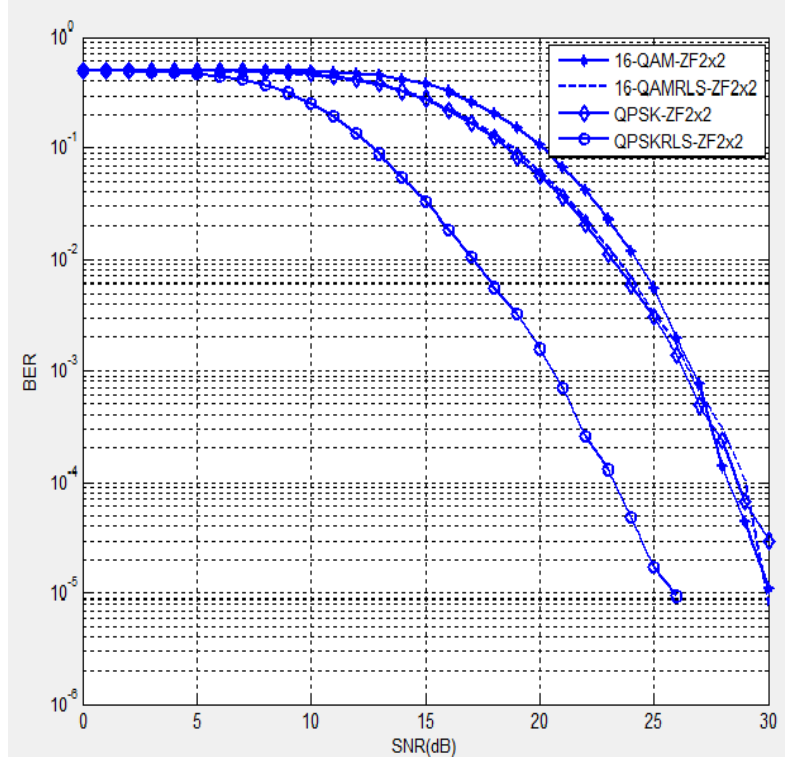

Fig 14: BER analysis for V-BLAST-ZF MIMO-OFDM With (RLS \& LMS) and Without Beamforming for 16QAM and QPSK Modulation using 2X2 Antenna 
Figure 14 explains the BER analysis of 16QAM and QPSK MIMO-OFDM system with RLS algorithm. VBLAST-ZF is used as detection algorithm and performance is measured with and without using adaptive Beamforming algorithm. It is observed that QPSK with RLS algorithm has better BER performance than others. Also, it can be observed that lower order modulation schemes have better BER performance over higher order modulation schemes.

\section{CONCLUSION}

In this paper adaptive Beamforming algorithms along with MIMO-OFDM have been studied, analyzed and compared with the help of simulation in the section 7. The plot is in between BER and SNR and hence compared the adaptive Beamforming algorithms with different spatial degree of MIMO. The plots are simulated in Rayleigh fading channel with V-BLAST-ZF/MMSE detection for MIMO-OFDM. MIMO-OFDM itself has the good spectral efficiency and by adding Beamforming algorithm the performance of the system can be further enhanced. From the paper, it can be concluded that the performance of V-BLAST MIMO-OFDM system is increased with Beamforming techniques. The system performance is better for RLS adaptive algorithm than that of LMS algorithm. The BER performance is good for QPSK modulation than 16QAM and finally as the number transmitter and receiver increases the BER performance of the system is also increased. Also the BER performance can be increased by using MMSE nulling than that of ZF nulling. In conclusion, BER performance of the MIMO-OFDM system with Beamforming can be enhanced by approximately $10 \mathrm{~dB}$.

\section{REFERENCES}

[1] Anubhuti Khare, Manish Saxena and Vijendra Singh Mandloi, "Performance Analysis of V-BLAST Based MIMO-OFDM System with Various Detection Techniques", International Journal of Engineering and Advanced Technology, Vol.1, Issue-2, December 2011.

[2] Shreedhar A Joshi, Dr.Rukmini T S, Dr. Mahesh H, "Analysis of V-BLAST Techniques for MIMO Wireless channels with different modulation techniques using Linear and Non Linear Detection", International Journal of Computer Science, Vol.1,Issue 1, November 2011.
[3] Dhruv Malik and Deepak Batra," Comparison of Various Detection Algorithms in a MIMO Wireless Communication Receiver", International Journal of Electronics and Computer Science Engineering, Vol. 1, No. 3, Page 1678-1685

[4] Sanjivan Satyal, "BER Performance of OFDM with GI in SISO and MIMO System" International Journal of Computer Applications (0975-8887) volume 104 - No 5, October 2014

[5] Najoua Achoura and Ridha Bouallegue, "Channel Estimation for MIMO-OFDM beamforming systems", IJCSNS,Vol.8,No.3,March 2008

[6] Gurpreet Singh, Priyanka Mishra and Rahul Vij, "BER Analysis of V-BLAST MIMO Systems under Various Channel Modulation Techniques in Mobile Radio Channels", International Conference on Computer Technology and Science, Vol. 47, 2012

[7] Zijian Wang, Wen Chen and Jun Li, "Efficient Beamforming for MIMO Relaying Broadcast channel with Imperfect Channel Estimation", IEEE Transections on Technology,Vol. 61,No.1,January 2012

[8] Bishwarup Mondal and Robert W. Health, "Performance Analysis of Quantized Beamfoming MIMO Systems", IEEE Transections on Signal Processing, Vol.54, No.12,December 2006

[9] Syed. Shameem and Dr. Habibullah Khan, "Performance Comparison of LMS, SMI and RLS Adaptive Beamforming Algorithms for Smart Antennas", International Journal of Computer Science and Telecommunication, Vol.3, Issue 2, April-June 2012.

[10] Ravi Kumar and Rajiv Saxena, "Capacity Analysis of MIMO Spatial Channel Model using Novel Adaptive Semi Blind Estimation Scheme", Journal of Wireless Networking and Communications Vol.2, Issue 4, Page 66-74, 2012 